\title{
ACUTE PSYCHOTIC PRESENTATION IN SYPHILIS - THE GREAT IMITATOR IS BACK
}

\author{
Bethan Clare ap Rees ${ }^{1}$, Mudasir Firdosi ${ }^{1,2} \&$ Mohammad Zia UI Haq Katshu ${ }^{3}$ \\ ${ }^{I}$ South West London and St George's Mental Health NHS Trust, London, United Kingdom \\ ${ }^{2}$ Queen Mary's Hospital, London, United Kingdom \\ ${ }^{3}$ Institute of Mental Health, University of Nottingham \& Nottinghamshire Healthcare NHS Foundation Trust, \\ Nottingham, United Kingdom
}

received: 28.1.2021;

revised: 13.2.2021;

accepted: 20.2.2021

$* \quad * \quad * \quad * \quad *$

\section{INTRODUCTION}

In the late $19^{\text {th }}$ and the early $20^{\text {th }}$ century, general paresis of the insane, as it was known historically, or neurosyphilis was a common cause of new-onset psychosis. Symptomatic neurosyphilis was reported in 10-20\% patients with syphilis (Singh \& Romanowski 1999). The widespread use of penicillin resulted in a marked reduction in syphilis (Kim 1975). Towards the end of the $20^{\text {th }}$ century, syphilis was considered a rare disease and its relevance in clinical training and practice in general, and in neuropsychiatric settings in particular, diminished (Ropper 2019). Unfortunately, there has been a global resurgence of syphilis over the past decade. In England, between 2010 and 2019, the number of newly diagnosed syphilis patients increased from 2646 to 7982 (Mitchell et al. 2020). A similar increase from 45844 to 115045 was observed in the USA between 2010 and 2018 (Centers for Disease Control and Prevention 2019). Despite these increasing numbers, the clinical interest in syphilis, known for its protean manifestations earning it the name of the 'great imitator', remains low.

\section{CASE REPORT}

A man in his fifties was referred for potential firstepisode psychosis following a six-month deterioration in his mental health. He was overfamiliar, hyperactive, circumstantial in his speech, and labile in mood, fluctuating from elation to tearfulness. He had grandiose (believed that he had links with secret services and was in possession of vital national security information), persecutory (believed that a cochlear implant had been inserted into his brain to control his thoughts, emotions and body), and referential delusions, and passivity phenomena. He was experiencing hallucinations in multiple sensory modalities - visual, auditory, and tactile. He was carrying knives with him due to his persecutory beliefs and was suicidal. He lacked insight into his mental illness, needing detention and treatment in hospital.

He had no previous history of mental illness or any relevant family history. He was single, unemployed, and identified himself as gay. He had a previous history of substance use (Gamma-hydroxybutyrate, GHB), but no recent exposure. He was diagnosed with Human Immunodeficiency Virus (HIV) six years ago and started on antiretroviral medications. He was diagnosed with tinnitus and hearing loss four years ago. Physical examination revealed mild paresis of the right lateral rectus muscle, nystagmus on horizontal left gaze, sluggish bilateral lower limb deep tendon reflexes and loss of vibration sensation below knees bilaterally. His cognitive assessment done using Addenbrooke's Cognitive Examination-III was normal, with a score of 97/100.

Routine blood investigations on admission, including full blood count, urea and electrolytes, liver function tests, bone profile, lipid profile and thyroid function, were normal. Serum HIV-1 viral load 3 months prior to admission was 23200 copies $/ \mathrm{ml}$, which on admission reduced to $310 \mathrm{copies} / \mathrm{ml}$ and further reduced to 81 copies/ml, 4 days into admission. This was thought to be due to poor concordance with antiretroviral medications. Syphilis serology testing done 8 months prior to admission had shown positive Treponema pallidum haemagglutination assay (TPHA) and rapid plasma reagin test (RPR) with a titre of 1:256, which is consistent with a diagnosis of syphilis. He had not had any treatment for syphilis following the positive serum sample due to poor engagement with the genitourinary clinic.

Cerebrospinal fluid (CSF) analysis following admission showed elevated protein of $67 \mathrm{mg} / \mathrm{dL}$ and positive Treponema pallidum particle agglutination (TPPA) assay, with a titre of 1:320. RPR was negative. CSF glucose (3.2 $\mathrm{mmol} / \mathrm{L}$ ), lactate dehydrogenase (LDH, 20 units/L), white blood cells $\left(<1 \times 10^{6} / \mathrm{L}\right)$, and red blood cells $\left(300 \times 10^{6} / \mathrm{L}\right)$ were all within normal ranges. HIV viral load was undetectable $(<140$ copies/mL). Viral polymerase chain reaction (PCR) for Herpes Simplex virus 1 and 2, Varicella Zoster virus, Epstein Barr virus, Adenovirus, Enterovirus, Parechovirus, Cytomegalovirus, Human Herpesvirus 6, and John Cunningham virus were negative. Acid fast bacilli (AFB) and yeast cultures were negative.

Neuroimaging (CT \& MRI of head) was unremarkable except for mild opacification mastoid air cells bilaterally.

A diagnosis of neurosyphilis (ICD-10 code A52.3) and organic delusional (schizophrenia-like) disorder (ICD-10 code F06.2) was made. Treatment with pro- 
caine benzylpenicillin 2,400,000 units intramuscular and probenecid $500 \mathrm{mg}$ oral four times a day for 14 days for neurosyphilis was administered. Olanzapine titrated up to $15 \mathrm{mg}$ daily for psychosis was started. His HIV treatment was continued - raltegravir $1200 \mathrm{mg}$ mane, emtricitabine $200 \mathrm{mg}$ / tenofovir alafenamide $25 \mathrm{mg}$ mane. His psychosis improved markedly with a reduction in Brief Psychiatric Rating Scale scores from 69 on admission to 45 in two weeks, and further reduction to 28 in six weeks after treatment. He developed some insight into his mental illness and had residual paranoia at the time of discharge from hospital.

\section{DISCUSSION}

Neuropsychiatric presentations have been reported in $17-52 \%$ patients with neurosyphilis; the wide variation partly reflects the differences in clinical settings. Cognitive impairment, personality changes and delirium are the most common reported presentations, particularly in the recent decades. However, the clinical presentations are protean and can mimic most neuropsychiatric disorders, including mania, depression, and psychosis, which were reported more often in the late $19^{\text {th }}$ and early $20^{\text {th }}$ century (Lin et al. 2014). This change in predominant clinical presentations has been partly attributed to the widespread use of antibiotics. Our patient presented with florid psychotic symptoms with largely preserved cognitive functions; pertinently he had not had any antibiotic treatment for syphilis prior to this presentation. Cerebral atrophy, demyelination and infarcts have been reported in neurosyphilis, however, often neuroimaging is normal, like in our patient (Friedrich et al. 2014).

The progression from primary infection to symptomatic neurosyphilis usually takes years. However, coinfection with HIV, particularly untreated, is known to accelerate this process; neurosyphilis is nearly twice as prevalent in patients with HIV than those without. This increased risk is thought to be mediated by impaired immune-mediated clearance of the spirochaete (Gonzalez et al. 2019). Our patient had HIV co-infection, was poorly concordant with HIV treatment, and had high HIV RNA titres prior to admission, which perhaps explains the rapid development of neurosyphilis.

There is limited evidence to support specific recommendations on the use of antipsychotic agents for the treatment of psychosis in neurosyphilis (Wright \& Zvartau-Hind 2011). Anecdotal reports support the use of both first- and second-generation antipsychotics, like haloperidol, olanzapine, risperidone and quetiapine, at the lowest effective dose, alongside antibiotics, and attempts to reduce when clinically feasible (Sanchez and Zisselman 2007). Considering the reemergence of syphilis, there is urgent need for research to build evidence-based practices on neuropsychiatric aspects of neurosyphilis and increased emphasis in clinical training and practice.

\section{Acknowledgements: None.}

\section{Conflict of interest: None to declare.}

\section{Contribution of individual authors:}

Bethan Clare ap Rees \& Mudasir Firdosi examined the patient and collected the clinical data.

Bethan Clare ap Rees undertook planning of the report, consented the patient, completed literature review.

Mudasir Firdosi \& Mohammad Zia UI Haq Katshu wrote the manuscript.

All authors were involved the final review and editing of manuscript.

\section{References}

1. Centers for Disease Control and Prevention: Sexually Transmitted Disease Surveillance 2018. U.S. Department of Health and Human Services, Atlanta, 2019

2. Friedrich F, Aigner M, Fearns $N$, Friedrich ME, Frey $R$, Geusau A: Psychosis in Neurosyphilis - Clinical Aspects and Implications. Psychopathology 2014; 47:3-9

3. Gonzalez H, Koralnik IJ, Marra CM: Neurosyphilis. Semin Neurol 2019; 39:448-455

4. Kim CK: A clinical study of neurosyphilis. J Korean Neuropsychiatr Assoc 1975; 15:119-25

5. Lin L, Zhang H, Huang S, et al.: Psychiatric manifestations as primary symptom of neurosyphilis among HIVnegative patients. J Neuropsychiatry Clin Neurosci 2014; 26:233-240

6. Mitchell H, Allen H, Sonubi T, Kuyumdzhieva G, Harb A, Shah A, et al.: Sexually transmitted infections and screening for chlamydia in England, 2019. Public Health England, London, 2020

7. Ropper AH: Neurosyphilis. N Engl J Med 2019; 381:13581363

8. Sanchez FM, Zisselman MH: Treatment of psychiatric symptoms associated with neurosyphilis. Psychosomatics 2007; 48:440-5

9. Singh AE, Romanowski B: Syphilis: review with emphasis on clinical, epidemiologic, and some biologic features. Clin Microbiol Rev 1999; 12:187-209

10. Wright $P$ \& Zvartau-Hind M: Core Psychiatry Book. 2011, 3rd Ed:391-420

\section{Correspondence:}

Dr Mudasir Firdosi MD, MRCPsych, Consultant Psychiatrist \& Honorary Senior Lecturer

South West London \& St George's Mental Health Trust and St George's, University of London

Queen Mary's Hospital, London SW15 5PN, United Kingdom

E-mail:mudasirfirdosi@gmail.com 